\title{
Case report and literature review of a rare diagnosis of ossifying renal tumour of infancy
}

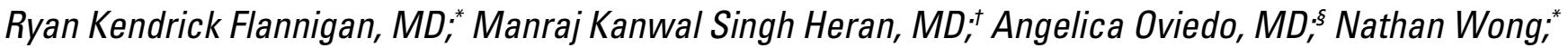 \\ John S.T. Masterson, MD, FRCSC*
}

*Department of Urological Sciences, University of British Columbia, Vancouver, BC; 'Department of Radiology, BC Children's Hospital, University of British Columbia, Vancouver, BC; $\$$ Department of Pathology, University of British Columbia, Vancouver, BC; * Department of Urological Sciences, Division of Pediatric Urology, BC Children's Hospital, University of British Columbia, Vancouver, BC

Cite as: Can Urol Assoc J 2014;8(3-4):e184-7. http://dx.doi.org/10.5489/cuaj.1454 Published online March 11, 2014.

\section{Abstract}

One must entertain a broad differential diagnosis for infants presenting with gross hematuria. Initial workup includes urine analysis, serum laboratory values and abdominal ultrasound. We describe an infant presenting with gross hematuria found to have a calcified renal mass upon initial ultrasound and subsequent computed tomography scan. We considered a differential diagnosis of, but not exclusive to, staghorn calculi, nephroblastoma, Wilms' tumour, mesoblastic nephroma and ossifying renal tumour of infancy (ORTI). A nephrectomy was performed, and the pathology report identified the calcified mass as an ORTI.

\section{Introduction}

There are a wide variety of renal neoplasms in the pediatric population. The differential diagnosis of a renal neoplasm ranges from more common entities, including neuroblastoma, nephroma and Wilms' tumour, to less frequent ones, such as renal cell carcinoma, and rare entities, such as ossifying renal tumour of infancy (ORTI). It is important to differentiate among different types of renal masses clinically, radiologically and pathologically as their treatment and follow-up differ.

ORTI is a rare, but likely benign, renal tumour, which was first described by Chatten and colleagues in 1980. ${ }^{1}$ To date, there are 16 reported cases. ${ }^{1-16}$ We describe a 4-month old boy with ORTI who was treated with a radical nephrectomy. At his 2-month follow-up, he was without recurrence.

\section{Case report}

We describe a case of a 4-month old boy who presented with painless gross intermittent gross hematuria since the age of 3 weeks. He had no signs of a urinary tract infection in either clinical or laboratory parameters. Urinalysis demonstrated 100+ red blood cells in high-powered field, and $1+$ protein; there was no evidence of a glomerular process. Renal function tests were well within normal limits and no electrolyte abnormalities were present. Abdominal ultrasound was initially negative; however, upon a serial follow-up it revealed a partially calcified appearing mass in the upper pole of the left kidney with no distortion of the renal contour (Fig. 1, part A). There was associated mild pelvicalyceal dilatation. No abnormalities were identified in the other kidney. Doppler examination showed a solitary central signal within the mass. Plain radiograph revealed flecks of dystrophic calcifications in the expected area of the left kidney in the retroperitoneal space. The likely diagnosis of ORTI was based on clinical background and sonographic features. Further characterization of the lesion was achieved by non-contrast and contrast enhanced computed tomography $(\mathrm{CT})$. A CT revealed a $2-\mathrm{cm}$ benign looking partially ossified renal mass within the upper pole of the left kidney with surrounding non-enhancing low attenuation area that was likely a soft tissue component of the mass (Fig. 1, parts $B, C$ and D).

Urologic evaluation was performed by 2 surgeons, with additional input from a pediatric oncologist. A partial nephrectomy was considered; however, given the spiculations extending from the mass on $\mathrm{CT}$, it was felt that enucleation and clear margins may not have been technically feasible. Thus, we decided to proceed with an open subcostal radical nephrectomy. Intraoperatively, no obvious gross extension of the tumour was identified and the procedure was conducted without complication. The patient had an uncomplicated hospital stay and was discharged on postoperative day 3. Pathology results returned with a diagnosis of ORTI, with the mass measuring $2.5 \times 2.1 \mathrm{~cm}$ (Fig. 2).

The peripheral margin of the mass was $1 \mathrm{~mm}$ from the renal capsule. Histology is demonstrated in Fig. 3 and Fig. 4.

At the 3- and 9-month follow-ups, the patient was clinically well with no recurrence of hematuria. He recovered 


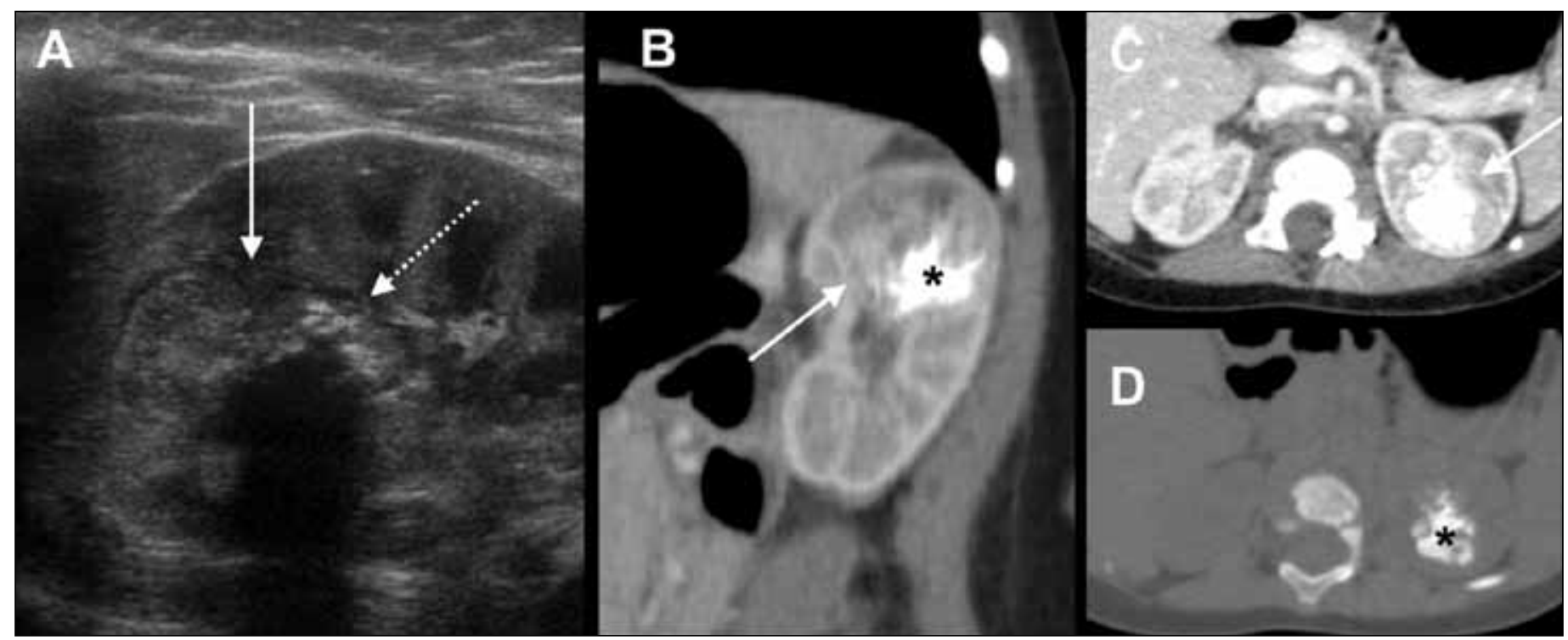

Fig. 1. Selected ultrasound image of the left kidney with a linear probe (A) demonstrates a well-circumscribed mass in the upper pole (solid white arrow), with internal high echogenicity with associated shadowing (dotted white arrow), representing the pathologic proven ossification within the tumour. Sagittal reformatted computed tomographic (CT) image (B), and axial CT image on soft tissue (C) and bone (D) windows again show the left upper pole mass (solid white arrow), with internal ossification (black asterisk).

from surgery. Repeat abdominal ultrasound was normal with no evidence of recurrence in the ipsilateral or contralateral sides.

\section{Discussion}

ORTI is a very rare, benign renal tumour. To date, there are 16 reported cases. ${ }^{2-8}$ Although rare, ORTI has certain epidemiological and radiological characteristics that may assist clinicians and radiologists in the diagnosis. The disease typically occurs during infancy, with patient age ranging from 6 days to 30 months. ${ }^{2,6}$ Of the 16 cases of ORTI, most were with boys $(75 \%, n=12)$. About $96 \%(n=15)$

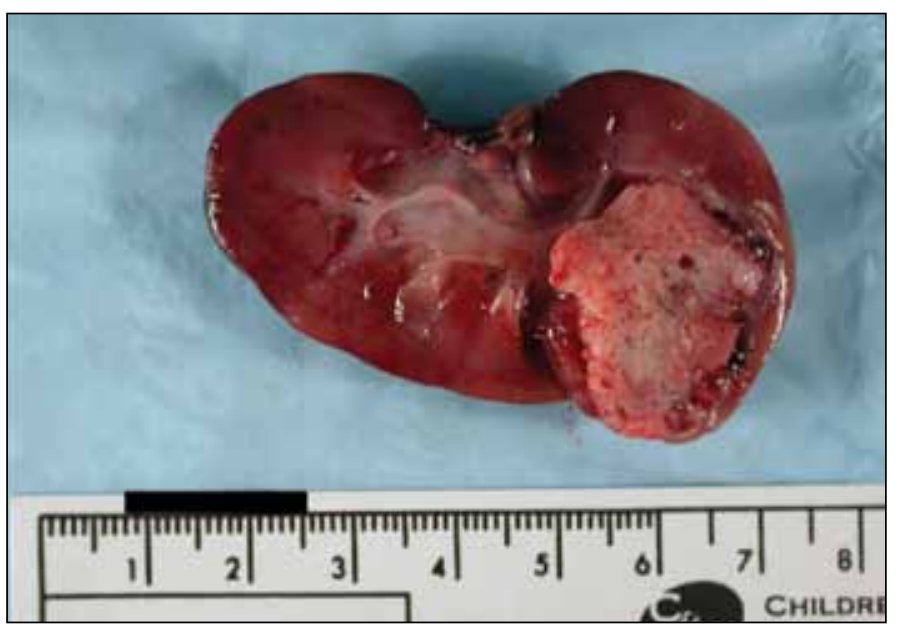

Fig. 2. Gross specimen, coronal section demonstrating a well-circumscribed $2.5-\mathrm{cm}$ lesion to the left upper pole. Surrounding parenchyma is unremarkable. of the patients presented with painless intermittent macroscopic hematuria. Although less frequent, an abdominal mass may also be present. ${ }^{8}$ Initial imaging investigations typically include ultrasound and CT. An ultrasound typically shows an echogenic mass with shadowing, while CT often shows a well-defined mass with calcification and poor enhancement. Most $(75 \%, \mathrm{n}=12)$ of the reported cases involved the left kidney, $69 \%(n=11)$ involved the upper pole calyces, and $81 \%(n=13)$ had calcification. The masses are rarely greater than 2 to $3 \mathrm{~cm}$ in size. Renal outline is typically maintained, and a filling defect with a local caliectasis is often seen. As our patient exhibited all

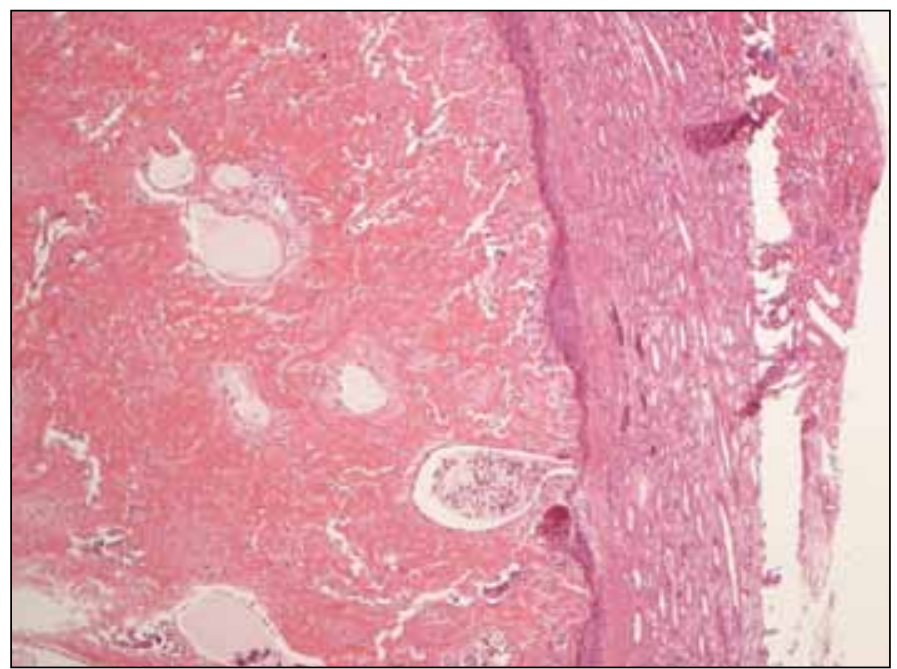

Fig. 3. Low power image demonstrates osteoblasts producing osteoid on the left. The blastemal cells and native kidney can be seen on the right. 


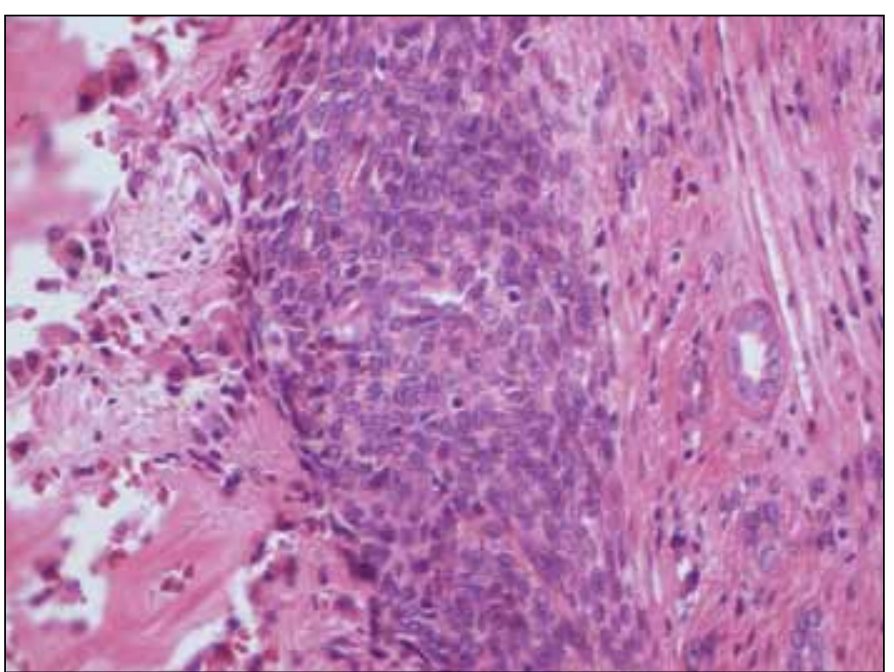

Fig. 4. High power of the edge of the tumour shows osteoid with osteoblasts on the left and mitotically active blastemal cells at the centre. Native renal parenchyma on the right.

of the above-described demographics, clinical and radiological features, the provisional diagnosis of ORTI was made preoperatively with a high degree of confidence.

Ossification, as seen with ORTI, may easily be mistaken for renal staghorn calculi. However, this diagnosis is exceedingly rare in infancy and thus alternative diagnoses should always be considered. Beyond the infancy period, making the diagnosis of ORTI can become more difficult because having a renal calculus is far more common than having ORTI. Nevertheless, ORTI presents with painless hematuria, and renal calculi are unlikely to be painless.

Other diagnoses on the differential for a calcified renal mass in this particular age group include Wilms' tumour, nephrogenic rest, renal cell carcinoma, extra-adrenal neuroblastoma, calcified hematoma and post-infectious (e.g., tuberculosis) or post-traumatic changes. ${ }^{5}$ Paraneoplastic syndromes, such as hypertension and hypercalcemia, could also be present in some of these disease entities. ${ }^{4}$

Histopathological diagnosis requires the presence of ossification of tissue. Typically, analysis demonstrates an osteoid core, layers of osteoblasts and an epitheilial component or spindle cells within the osteoid core. Mature osteoid elements tend to be more prominent in older patients. Despite clear visual characteristics, histiogenesis is not well-established for this unusual neoplasm. The origin of the tumour remains unclear, but some believe it arises from the urothelium. ${ }^{2}$ Ossification can be the result of the osteogenic potential of the urothelial cells. ${ }^{1,2}$ The ossification and mesenchymal components are similar to mesoblastic nephroma histologically. ${ }^{5,10}$ The mass is described as congruent with the renal medulla, in particular the papillae of the renal pyramids. From this location, it extends into the collecting system in a polypoidal manner. A competing theory suggests that spindle cells resemble intralobar nephrogenic rests and that these lesions may lie within the pathologic spectrum of Wilms' tumours. However, to date, Wilms' tumour has not been reported in any of these patients. ${ }^{2}$

No known associations with this tumour have been identified. However, Seixas-Mikelus and colleagues described a 3-month-old male infant with gross hematuria and ORTI in addition to juvenile granulosa cell tumour of the testis. This is the only case report of these 2 lesions presenting concomitantly. ${ }^{7}$ The case likely represents a chance finding of 2 distinct disease processes. A potentially newly discovered association cannot be made based on a single case; it requires a more scientific analysis of the disease processes. El-Husseini and colleagues reported a 10-month-old girl with ORTI and asthma. ${ }^{3}$ However, this was likely an incidental finding and not related to ORTI.

Although it is considered benign, the true natural history is still a mystery as all reported cases have been treated with surgery, with partial or total nephectomy. However, Stefeens and colleagues have reported that complete enucleation of the mass without nephrectomy or heminephrectomy may be an alternative treatment strategy. In addition, the use of chemotherapy is not justified, as patients who were only treated surgically had no evidence of recurrence or metastases. The longest follow-up reported was by Ito and colleagues, who had a follow-up of 23 years with no recurrence. Thus, it is important to correctly diagnose ORTI to avoid any unnecessary treatment. However, since only 16 cases have been described, no absolute conclusions may be drawn. Thus, patients should always be followed clinically and with postoperative imaging, such as ultrasound.

\section{Conclusion}

ORTI is a very rare renal entity that has some typical clinical, radiological and pathological features. Sixteen reported cases are not significant enough to draw any specific conclusions. However, the current data suggest that a male infant with painless intermittent macroscopic hematuria, with radiological features of a non-invasive calcified renal mass within the upper pole calyceal system, very likely has an ORTI. This constellation of clinical presentation and radiologic findings strongly supports the diagnosis of ORTI prior to pathologic confirmation.

Competing interests: Dr. Flannigan, Dr. Heran, Dr. Oviedo, Dr. Wong and Dr. Masterson all declare no competing financial or personal interests.

This paper has been peer-reviewed. 


\section{References}

1. Chatten J, Cromie WJ, Duckett JW. Ossifying tumor of infantile kidney: Report of two cases. Cancer 1980;45:609-12

2. Lowe LH, Isuani BH, Heller RM, et al. Pediatric renal masses: Wilms tumor and beyond. Radiographics 2000;20:1585-603. http://dx.doi.org/10.1148/radiographics.20.6.g00nv051585

3. El-Husseini TK, Egail SA, Al-Orf AM, et al. Ossifying renal tumor of infancy. Saudi Med J 2005;26:1978-9.

4. Glick RD, Hicks MJ, Nuchtern JG, et al. Renal tumors in infants less than 6 months of age. J Pediatr Surg 2004;39:522-5. http://dx.doi.org/10.1016/i.jpedsurg.2003.12.007

5. Höglund HHE, Kellner MW, Körber F, et al. Ossifying renal tumor of infancy (ORTI): A rare diagnosis. Klin Padiatr 2011;223:178-9. http://dx.doi.org/10.1055/s-0031-1275350

6. Schelling J, Schröder A, Stein R, et al. Ossifying renal tumor of infancy. J Pediatr Urol 2007;3:258-61. http://dx.doi.org/10.1016/i.jpurol.2006.05.009

7. Seixas-Mikelus $S A$, Khan A, Williot PE, et al. Three-month-old boy with juvenile granulosa cell tumor of testis and ossifying renal tumor of infancy. Urology 2009;74:311-3. http://dx.doi.org/10.1016/j.urology.2008.10.055

8. Vazquez JL, Barnewolt CE, Shamberger RC, et al. Ossifying renal tumor of infancy presenting as a palpable abdominal mass. Pediatr Radiol 1998;28:454-7. http://dx.doi.org/10.1007/s002470050381
9. Ito J, Shinohara N, Koyanagi T, et al. Ossifying renal tumor of infancy: The first Japanese case with longterm follow-up. Pathol Int 1998;48:151-9. http://dx.doi.org/10.1111/i.1440-1827.1998.tb03885.x

10. Fernbach SK, Schlesinger AE, Gonzalez-Crussi F. Calficiation and ossification in a congenital mesoblastic nephroma. Urol Rad 1985;7:165. http://dx.doi.org/10.1007/BF02926878

11. Steffens J, Kraus J, Misho B, et al. Ossifying renal tumor of infancy. J Urol 1993;149:1080-1.

12. Caillet M, Massot, Taillard. A case of calcified nephroblastoma simulating a calculus. I Urol Nephrol 1967;73:653-5.

13. Charles AK, Berry PJ, Joyce MR, et al. Ossifying renal tumor of infancy. Pediatr Pathol Lab Med 1997:17:322-4.

14. Jerkins GR, Callihan TR. Ossifying renal tumor of infancy. J Urol 1986;135:120-1.

15. Sotelo-Avila C, Beckwith JB, Johnson JE. Ossifying renal tumor of infancy: A clinicopathologic study of nine cases. Pediatr Pathol Lab Med1995;15:745-62. http://dx.doi.org/10.3109/15513819509027010

16. Middlebrook PF, Jimenez CL, Schillinger JF. Ossifying Renal Tumor of infancy: A case report. J Urol 1992; 147:1337-9.

Correspondence: Dr. Ryan Kendrick Flannigan, Department of Urological Sciences, University of British Columbia, Vancouver, BC; rkflanni@gmail.com 